\title{
Clock-like Flow Replacement Schemes for Resilient Flow Monitoring
}

\author{
Gunwoo Nam, Pushkar Patankar, Seung-Hwan Lim, Bikash Sharma, George Kesidis, and Chita R. Das \\ Department of Computer Science and Engineering \\ The Pennsylvania State University, University Park, PA 16802 \\ Email: \{gnam, patankar, seulim, bus145, kesidis, das\}@cse.psu.edu
}

\begin{abstract}
In the context of a collaborating surveillance system for active TCP sessions handled by a networking device, we consider two problems. The first is the problem of protecting a flow table from overflow and the second is developing an efficient algorithm for estimating the number of active flows coupled with the identification of "heavy-hitter" TCP sessions. Our proposed techniques are sensitive to limited hardware and software resources allocated for this purpose in the linecards in addition to the very high data rates that modern line cards handle; specifically we are interested in cooperatively maintaining a per-flow state with a low cost, which has resiliency on dynamic traffic mix. We investigate a traditional timeout processing mechanism to manage the flow table for per-flow monitoring, called Timeout-Based Purging (TBP), our proposed Clock-like Flow Replacement (CFR) algorithms using a replacement policy, called "clock", and a hybrid approach combining these two. Experiments with Internet traces show that our CFR schemes can significantly reduce both false positive and false negative rates, regardless of whether the flow table is fully occupied (even under SYN flooding) or sufficiently empty. Our hybrid scheme estimates the number of active flows accurately, and confines the heavy-hitters without storing packet counters.
\end{abstract}

\section{Introduction}

Modern networking devices have the capability of monitoring tens of thousands of TCP sessions [1], [2]. The goal of such monitoring could be to detect attacks or intrusions or to deter end-systems that opt-out of communal (and voluntary) TCP congestion control mechanisms. Traffic monitoring, QoS assurance and usage-based billing [3] also motivate perflow monitoring of TCP flows.

The overhead of maintaining the per-flow monitoring in network devices, i.e., wire-speed packet processing and corresponding updates [4], is not trivial. A large number of short-lived flows or abnormal flows results in significant consumption of the flow memory as well as an increase in the volume of data exchanged for collaboration. Even though

This work is supported by a Cisco Systems URP gift and by the NSF under grant CyberTrust 0524202. a SRAM implementation of the flow table has become typical for fast operations despite its limited capacity [5], [6], this has worsen the scaling problem. Since prediction of the "proper" size of flow table is not trivial, the per-flow monitoring should adapt to the dynamic traffic conditions. Under the a flash crowd of short-lived flows or Denial-ofService (DoS) attacks the per-flow monitor cannot preserve normal flows correctly or memory resource might be exhausted in an extreme situation [7]. For this reason, the perflow monitoring system itself could be targeted by malicious attacks, e.g., SYN flooding or port scanning, which could result in performance degradation of the networking device and the network connected to it [8], [9]. To remove or disable entire set of stale (not recently touched) flows from memory, time-outs can generally be managed to evict outdated flows using a "touch bit" at the flow level [5], [10]. However, a time-out threshold [11], [8] has a trade-off in protecting flow memory and prematurely purging flows. If time-out is too short, it is less vulnerable to malicious attacks but active flows could be falsely removed. Conversely, If time-out is too long, it increases the number of flow entries stored in the flow table.

This complex performance and scalability issues motivate us to devise a method to fully utilize the flow table regardless of traffic conditions, i.e., protect routers maintaining perflow state from flow table overflows in the presence of SYN floods (or also flash crowds) and preserve as many idle flows as the maximum capacity of the flow table permits. To achieve this, we suggest a low cost flow replacement scheme for maintaining per-flow state. First, we investigate a Clocklike Flow Replacement (CFR) scheme employing a simple and fast replacement policy, called "clock" [12]. In CFR, flows are replaced based on the touch bit indicating frequency of incoming packets. It performs similar to the Least Recently Used (LRU) algorithm, without the overhead of maintaining the sorted list of flows. By extending the touch bit to two bits, we can discriminate several groups of flows based on the number of incoming packets as SYN packets, short-lived flows and long-lived flows. The multiple bits allows multi-level replacement based on clustering, which makes the system scan-resistant to port scanning or SYN flooding [12]. We then investigate a hybrid approach, called Clock-like Flow Replacement with Lazy Purging (CFR-LP), combining CFR and a traditional purging scheme. CFR-LP 
reduces the volume of the flow table by removing outdated flows periodically.

We evaluate the performance of our algorithms using three sets of Internet traces collected from different networks. We compare their performance with two state-of-the-art algorithms, namely Finger-Compressed Filter (FCF) [5] and Timeout Based Purging (TBP). To keep the comparisons fair, we restrict our simulation study to only those methods that maintain per-flow state.

Our experimental results indicate that our CFR-LP exhibit substantially better performance in important performance metrics: False Positive Rate (FPR), False Negative Rate (FNR) and estimation of the average number of active flows. CFR-LP reduces false positive and false negative rates as compared to FCF by $78 \%$ and $73.7 \%$, and it outperforms TBP by $51.1 \%$ and $12.5 \%$ when the table size is twice the average number of active flows. These tendencies were steadily observed even under SYN flooding regardless of the load on the flow table. In particular, this CFR-LP showed the best accuracy in estimating the number of active flows. Finally, our CFR-LP can also confine heavy-hitters to a particular state without counting the number of packets. Our experiments showed that detected heavy-hitters are less than $10 \%$ of active flows with an error rate of $0.5 \%$.

The rest of this paper is organized as follows: In Section 2, we discuss related work. Our basic CFR algorithms and the hybrid technique is described in Sections 3 and 4. Then, implementation issues of our schemes are discussed in Section 5. Experimental results are given in Section 6, followed by conclusions in Section 7.

\section{Related Work}

Purging schemes, such as [5], [13], use a touch bit to purge stale flows. In these schemes, one touch bit is used to check a flow's activeness during the measurement time interval $T_{\theta}$. Incoming packets modify the corresponding flows' touch bit. Thus, at the end of every time interval $T_{\theta}$, flows whose touch bits were not activated may be purged. This scheme may require traversal of all the flow entries at the end of every timeout interval. We denote such schemes as Timeout Based Purging (TBP) methods.

A problem of TBP is how to set up the timeout threshold value without causing flow table explosion or prematurely purging active flows. Figure 1 shows the cumulative distribution function of maximum inter-packet time per flow for three Internet traces: SMTP, HTTP, MIXED [14], [15]. From this figure, we can see the relationship between a timeout threshold and the number of prematurely purged flows. For instance, if a timeout threshold $T_{\theta}$ is 30 seconds, up to $20 \%$ of flows may be wrongly purged, i.e., false negatives, while $T_{\theta}=120$ prematurely purges around $3-7 \%$ of active flows. In current networking devices [1], [16], 3600 seconds is used to expire established TCP connections. Even though

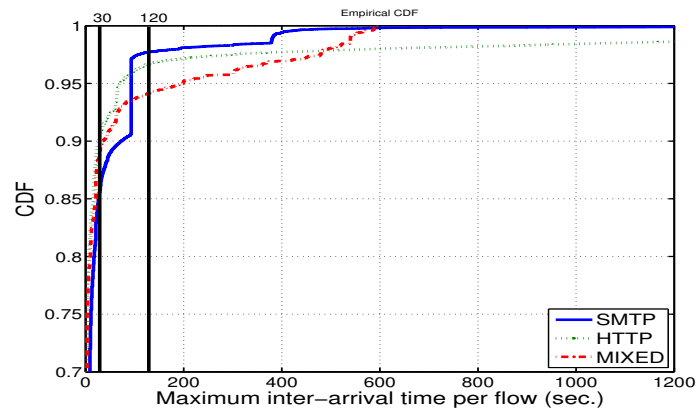

Figure 1. Maximum inter-packet time per flow

this greatly reduces false negatives, it requires a huge-sized flow table. NetFlow [17] uses 15 seconds, while 60 seconds is used in Adaptive NetFlow [18]. These various settings of the timeout threshold implies that choosing a proper timeout value is not straightforward, motivating us to investigate the CFR algorithms. Calibrating TCP session-level time-outs is explored in [8], [11]. Kim et al. [8] demonstrated that a long time-out threshold under SYN flooding results in a session table explosion. They only discussed the problem for the initial stage of a TCP session, but this kind of timeout mechanism experiences such trade-offs throughout the entire session lifetime.

A feasible and efficient management of finite-state machines (FSMs) monitoring active flows is discussed in [5], where each FSM (ACSM in [5]) approximates a single TCP session's state. They proposed the Fingerprint compressed Filter (FCF) based on $d$-left hashing and use a single "touch" bit for timeout, but it is a bucket-level purging rather than a flow-level purging. Similarly, [10] discussed the significance of massive purging overhead and introduced a late purging concept. While a hash chain corresponding to the flow ID of the incoming packet is searched in the flow (hash) table, outdated flows in the hash chain are checked to be purged. Both the above techniques, however, may give rise to high false positives, thereby overestimating the number of active flows.

[7] proposed a coordinated router-based flow monitoring, consisting of routers as monitor nodes for performing hashbased flow selection. It reduces the data redundancy of reported flow data, but as was mentioned, router memory could be exhausted in a node unless more sophisticated purging policies are used. Our replacement schemes, if implemented in these nodes, would allow a real-time collaborative flow monitoring.

Flow sampling approaches investigated the dynamic sampling rate to avoid a biased estimation, but it is not trivial to estimate the number of active flows in [18], [19], [20]. [19], [21], [22] propose techniques to detect heavy-hitters and estimate the number of active flows without maintaining per-flow state. 


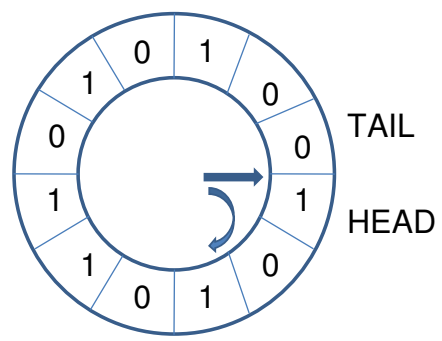

Figure 2. Concept of the "clock" algorithm

\section{A Clock-like Flow Replacement Policy}

In this section, we describe the Clock-like Flow Replacement (CFR) algorithm and present some improvements on it. We assume there is a per-flow monitoring system for bidirectional traffic in a linecard. Specifically, we consider a flow table consisting of Flow ID, touch bit, and state. To enable per-flow monitoring in high-end routers, the use of fast memory, such as SRAM, is required. We assume that the flow table is implemented using a hash table with hashchaining (We do not address the problem of hash collisions in this paper. Papers have referred to this problem, e.g., Shared Fast Hash Table [23], FCF [5], or a TCAM-based approach [9]. These approaches can be combined with our replacement schemes.). A flow ID is composed of several fields of the TCP and IP header, typically including the following 5-tuple (src ip, dst ip, src port, dst port, ip proto) [24]. Note that for a hashed flow ID, 32 bits may be employed to reduce the flow entry size. However, there is a trade-off between the size of the flow ID and hash collisions [9]. As a part of state information, packet counters may be a burden in a compact SRAM implementation, so the counters could be stored in DRAM as in [7], [23]. However, these implementations may slow wired-speed packet processing. Hence, our schemes employ probabilistic discrimination of flows based on the frequency of packets without explicitly storing packet counters.

\subsection{CFR}

CFR is stimulated by the "clock" [12], a page replacement algorithm, so that the flow table also can benefit from replacement free from overflow. The "clock" concept is simple to implement and performs similarly to the LRU cache algorithm in terms of page hit replacement, but with much reduced operational complexity by eliminating the need of maintaining an ordered list. In "clock", as shown in Figure 2, cached pages are maintained by using a circular buffer, called a clock, and each page has a page reference bit (a touch bit in our algorithm) indicating its reference. If a page hit occurs, the page reference bit is set to 1 . If a page miss occurs, then an evicted page is selected as follows: look at the current page under the HEAD pointer of the buffer

\begin{tabular}{|c|c|c|}
\hline State & Touch bits & Flow type \\
\hline$V_{0}$ & 00 & unused \\
\hline$V_{1}$ & 01 & initial state \\
\hline$V_{2}$ & 10 & short-lived \\
\hline$V_{3}$ & 11 & long-lived \\
\hline
\end{tabular}

Table 1. Touch bit states in CFR-2

and check its page reference bit. If the page reference bit is 1 , set it to 0 , move forward in clockwise rotation, until a page with reference bit 0 is encountered. This entry is then selected (a victim), and replaced with a new page reference.

In CFR, the touch bit is not set based on timeout, but depends on the "clock" look-up. For this, an additional circular list, a clock, is needed to maintain the order of replacement. This linked list could be a part of flow entry in the flow table or could be maintained separately. One of the advantages of the CFR algorithm is that the table overflow does not occur even when the flow table becomes full. When the flow table is full and all the entries' touch bits are 1, the algorithm traverses once through the clock, resetting all flows' touch bits to 0 , and finally visits the HEAD entry of the clock again and evicts the flow because its touch bit was set to 0 in the previous pass. The obvious disadvantage is that CFR may incur a significant overhead when trying to find a victim entry. For this purpose, we discuss a bitmap implementation and a provisioning lookup to decrease the lookup time in Section 5.

\subsection{CFR-2}

Extending the touch bit indicator in the previous algorithm to two bits allows us to discriminate flows according to the four possible states namely, unused flows, SYN flows, short-lived flows and long-lived flows. Table 1 shows the combination of touch bits and the corresponding flow states in CFR-2. This discrimination/clustering enables the monitoring system to report the data of only a particular state's flows and also in summarizing data of the interested clusters. The touch bits are toggled according to the frequency of packets as follows:

- If the flow "misses" in the flow table, i.e., if the flow ID of an incoming packet does not match an entry in the flow table, find a victim entry, using the "clock" mechanism as described below, and replace it with a new flow, setting its touch bits to 01 .

- If the flow "hits", update the entry and increment its touch bits by 1 with a probability $p_{1}$ or $p_{2}$ unless the touch bits of this flow are 11. Upon receipt of a RST or FIN packet, the touch bits are reset to 00 .

To instantiate a new flow in the flow table, the "clock" mechanism is executed to find a victim entry to be replaced as follows. The HEAD pointer moves sequentially across the flows decrementing their touch bits by 1 until it comes 


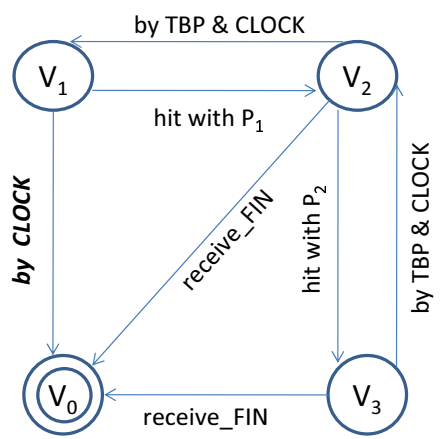

Figure 3. State transition diagram in CFR-LP

across a flow whose touch bits are set to 00 . This is the flow which is then replaced by the new flow.

Here, we adopt two probabilities $p_{1}$ and $p_{2}$ to toggle the touch bits of the flows in states $V_{1}$ and $V_{2}$, respectively. For example, if a flow $i$ is new, it starts with state $V_{1}$, i.e., its touch bit are set to 01 . If $p_{1}=1$ and $p_{2}=0.125$, the arrival of second packet of flow $i$ sets its touch bit to 10 . On an average, one packet among the next eight packets of flow $i$ will makes the flow's state transition from $V_{2}$ to $V_{3}$. Since long-lived flows have more than 10 packets [25], setting $P_{2}=0.125$ will restrict most long-lived flows to state $V_{3}$. Note that, this scheme does not rely on a specific purging mechanism for flow replacement. Flow entries are invalidated when its touch bits are set to 00 as a result of "clock" activity. This avoids the overhead of relying on an explicit purging scheme to remove outdated flows.

In this scheme, the touch bits (and its state) can give multilevel priority for the flow removal. Every state approximates the number of packets received for that flow without having to explicitly maintain the packet counts. For example, flows in state $V_{2}$, on an average, indicate that these flows have received less than 10 packets so far. State $V_{3}$ indicates that the flows in this state are typically long-lived flows. By decreasing the probabilities $p_{1}$ and $p_{2}$, we can confine heavyhitters to state $V 3$ thereby, separating them from normal flows. Selecting the probabilities $p_{1}$ and $p_{2}$ is a engineering issue which we will discuss in the context of heavy-hitter detection in Section 5.

When a new flow is inserted into the flow table, it is assigned state $V_{1}$ rather than $V_{2}$. When the flow table is full, flows in state $V_{1}$ are the first to be evicted, thereby protecting the flow table from contamination by spoofed SYN packets. Similar to SYN cache [26], this mechanism can also initially assign a limited amount of resources to SYN flows which can then be increased if the flow extends beyond SYN packets

\section{A Hybrid Technique}

In CFR and CFR-2, only "clock" activity can remove stale flows, thereby increasing the time duration required to find a

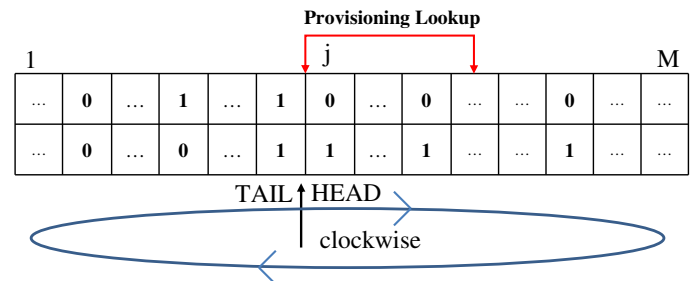

Figure 4. A bitmap implementation of CFR algorithms

victim entry. Also, both CFR and CFR-2 cannot estimate the number of active flows, because idle flows remain in the flow table until the flows are replaced out. Therefore, we need a scheme which correctly estimates the number of active flows with reduced memory accesses. Hence, we propose a hybrid technique which combines CFR-2 and TBP, called a Clocklike Flow Replacement with Lazy Purging (CFR-LP) in this section.

Figure 3 shows the state transitions of the touch bits in CFR-LP. Just as in CFR-2, this method relies on "clock" to decrement touch bits. However, it also incorporates a TBP mechanism which decrements the touch bits of flows in state $V_{2}$ and $V_{3}$ by 1 at the end of every timeout interval. An explicit purging mechanism can reduce the time duration for which stale flows are kept in the flow table. Hence, the "clock" activity can change the state of flows from $V_{1}$ to $V_{0}$ in CFR-LP, while the purging activity does not change the state of flows in $V_{1}$. As a result, idle flows are removed only when the flow table is full, thereby maximizing the utilization of flow table which increases an estimate of numbers of active flows. Since the flows are only purged as a result of "clock" activity, purging is delayed, hence the name "lazy" purging. In addition, flows only in $V_{1}$ and $V_{2}$ are regarded as active in CFR-LP to avoid overestimation of active flows due to multiple timeout intervals.

\section{Implementation Issues}

In this section, we discuss a bitmap implementation to reduce the memory overhead to maintain the circular buffer clock and then detect heavy-hitters.

\subsection{A bitmap implementation}

Considering the cost implications of using a smaller SRAM, the flow table maintained in a SRAM also needs to be small. Instead of maintaining the circular buffer clock, which takes up more memory, we can implement our scheme using a bitmap algorithm, consisting of an $M$ array of 2 bits. Figure 4 shows a bitmap implementation of CFR algorithms. "clock" like behavior can be achieved by simply adopting the modulo operation.

With a bitmap algorithm, we can significantly reduce the overhead of finding a victim entry along the clockwise 


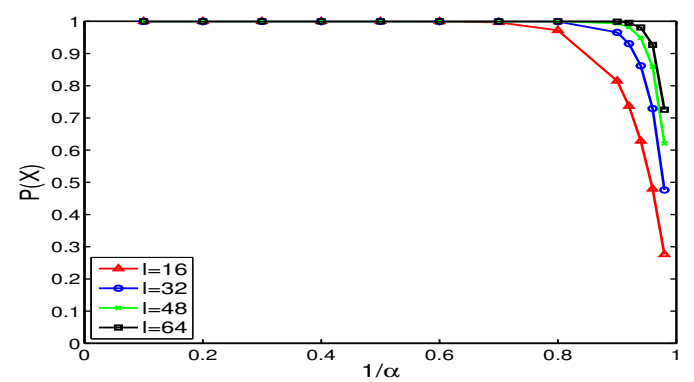

Figure 5. Lookup overhead in bitmap implementation

direction. To find a victim entry from the bitmap, we can fetch a word extending from $H E A D$ in one memory access, which has a 16 entries of two bit touch bits. We term this word provisioning lookup. Then, we can choose a first unused entry from the fetched word.

Preliminary Analysis: We assume that a flow table of size $M$ has three types of entries: flows in state $V_{2}$ and $V_{3}$, flows in state $V_{1}$, and unused entries in state $V_{0}$. The number of flows in state $V_{2}$ and $V_{3}$, flows in state $V_{1}$ and unused entries are defined as $a, p$ and $u$, respectively. Then, $M=a+p+u$. Let $l$ be the number of entries in a provisioning lookup. $X$ is the event that there is at least one unused entry in a provisioning lookup.

$$
\begin{aligned}
P(X) & =1-\frac{\left(\begin{array}{c}
a+p \\
l
\end{array}\right)}{\left(\begin{array}{c}
M \\
l
\end{array}\right)} \\
& =1-\left(\frac{1}{\alpha}\right)^{l} \cdot \frac{M \cdot(M-\alpha) \cdots(M-\alpha(l-1))}{M \cdot(M-1) \cdots(M-(l-1))} \\
& \geq 1-\left(\frac{1}{\alpha}\right)^{l},
\end{aligned}
$$

where $a+p=\frac{M}{\alpha}\left(l \leq \frac{M}{\alpha} \leq M\right)$.

Note that $\frac{1}{\alpha}=\frac{a+p}{M}=\frac{M-\bar{u}}{M}(\leq 1)$ indicates the fraction of used entries in the flow table. Based on equation (1), Figure 5 shows that the relationship between $P(X)$ and $\frac{1}{\alpha}$ for varying $l$. For example, if we let $\frac{1}{\alpha}=0.8$, then $u=0.2 M$ i.e., unused entries are $20 \%$ of the flow table. In a setting of $l=16$ and $M=1,000,000, P(X)=97.19 \%$.

Figure 5 indicates that the provisioning lookup reduces the "clock" overhead significantly. Our preliminary analysis shows that finding an unused entry requires only one memory access in most cases. FCF takes at least $d$, the number of hash functions (usually three or four), times of memory accesses [5]. Hence provisioning lookup is capable of efficiently maintaining the flow table in highspeed networking devices.

\subsection{Confining heavy-hitters}

By dynamically adjusting the probabilities $p_{1}$ and $p_{2}$ according to the current traffic conditions, we can confine heavy-hitters to state $V_{3}$. If we let $P_{\theta}$ and $N_{\theta}$ be the total

\begin{tabular}{|c|c|c|}
\hline \multirow{2}{*}{ Test result } & \multicolumn{2}{|c|}{ Actual condition (flow existence) } \\
\cline { 2 - 3 } & $\begin{array}{c}\text { Present } \\
\text { (flow existence) }\end{array}$ & $\begin{array}{c}\text { Absent } \\
\text { (flow removal) }\end{array}$ \\
\hline $\begin{array}{c}\text { Positive } \\
\text { (Test shows "existence") }\end{array}$ & $\begin{array}{c}\text { True positive (TP) } \\
\text { (active flows) }\end{array}$ & $\begin{array}{c}\text { False positive (FP) } \\
\text { (purged flows at next } \mathrm{T}_{\Phi} \text { ) }\end{array}$ \\
\hline $\begin{array}{c}\text { Negative } \\
\text { (Test shows "removal" ) }\end{array}$ & $\begin{array}{c}\text { False negative (FN) } \\
\text { (prematurely purged flows) }\end{array}$ & $\begin{array}{c}\text { True negative (TN) } \\
\text { (purged or closed flows) }\end{array}$ \\
\hline
\end{tabular}

Table 2. Relationships in binary classification

number of packets and the estimated number of active flows during the time interval $T_{\theta}$ respectively, the fair share of an individual flow will be $P_{\theta} / N_{\theta}$. When the total number of packets for a flow is greater than $n P_{\theta} / N_{\theta}$, for some $n>1$, the TCP flow can be identified as a heavy-hitter. Then, we can confine heavy-hitters to the state $V_{3}$ by adjusting $p_{1}$ and $p_{2}$, using the following equation:

$$
p_{1} \times p_{2}=\frac{N_{\theta}}{n \times P_{\theta}} .
$$

For example, if $P_{\theta}=10,000$ and $N_{\theta}=100$, then the average number of packets per flow is 100 . If we define $n=5$, then $p_{1} * p_{2}=0.05$. If we have a simple setting of $p_{1}$, say $p_{1}=1$ (i.e., any packet of a flow updates its state in $V_{1}$ to $V_{2}$ ) then $p_{2}$ can be set to 0.05. $P_{\theta}$ and $N_{\theta}$ can be recorded for each time interval and act as an input to the next phase which further causes an adjustment of the probabilities $p_{1}$ and $p_{2}$, thereby tuning them according to current traffic conditions.

Simulation results indicate that our hybrid scheme are able to restrict heavy hitters to state $V_{3}$ without having to explicitly maintain packet counters. Note, that these schemes are an approximate estimation just like [27], [28], [29], [30], since they are based on probabilistic methods and may not guarantee accurate estimation of heavy hitters. However, as compared to more accurate detection methods such as [31], our approaches incur considerable less overhead and can be used as a front end. Also, state-dependent update, e.g., sampling-based update of flows only in $V_{3}$, might help to further reduce monitoring overhead. Hence, our lightweight schemes can also be used for congestion control and fair queueing such as [13].

\section{Experimental Evaluation}

We use three objective metrics: False Positive Rate (FPR), False Negative Rate (FNR) and the number of active flows, to analyze our CFR algorithms and compare them with TBP [13] and FCF [5].

\subsection{Performance Parameters}

6.1.1. FPR and FNR. False positives, in the context of perflow monitoring, are those flows which could have been 


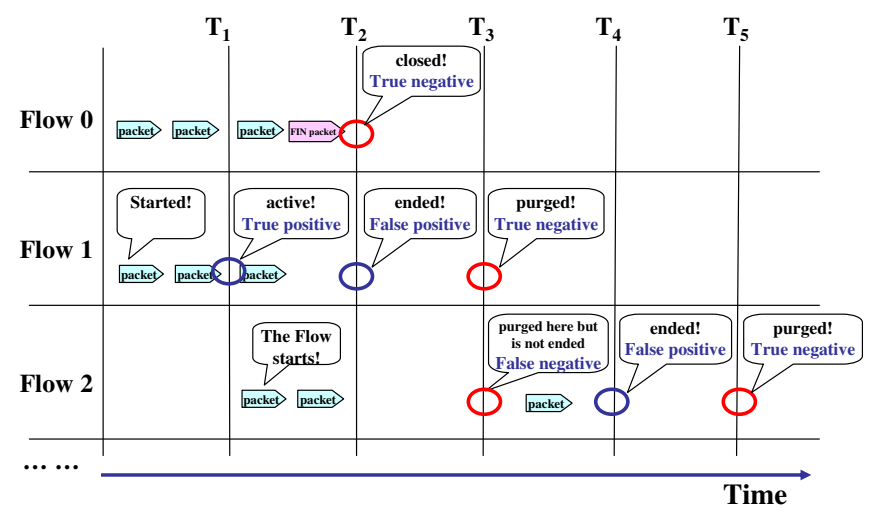

Figure 6. FPs and FNs in TBP

deleted but are not deleted, while false negative indicates flows which should not be deleted but are deleted, as shown in Table 2. One subtle thing regarding false negatives using TBP is the case when there is no available entry for new flows. In this case, this new flow is dropped in our experiments and, as a result, a false negative is added, just as in [7]. Therefore, in TBP, when the number of active flows is larger than the flow table size, the number of false negatives increases dramatically. The FPR and FNR are expressed as:

$$
F P R=\frac{F P}{F P+T N}, F N R=\frac{F N}{T P+F N} .
$$

6.1.2. Estimation of active flows. Flow termination by timeout gives rise to an imperfection in the estimation of the number of active flows as follows. As shown in Figure 6 , consider flow 1 , which sent it's last packet between the interval of $T_{1}$ and $T_{2}$ after which it went idle. The TBP mechanism cannot identify that the flow needs to be purged after time interval $T_{1}$ thereby, introducing a False positive at $T_{2}$ and a True negative at $T_{3}$. Hence, TBP overestimates the number of active flows at interval $T_{2}$. In a more complicated scenario in the case of flow 2, when the flow resumes sending its packet just past $T_{3}$, a false positive which was calculated at $T_{2}$ and a true negative which was calculated at $T_{3}$ should be invalidated. Hence, we can drive an equation to compute the number of active flows (called $N_{\theta}$ ) at the end of measurement interval $T_{\theta}$ as follows:

$$
N_{\theta}=T P_{\theta}+F N_{\theta}-F P_{\theta} .
$$

\subsection{Experimental Setup}

To investigate the performance of the proposed schemes, we conducted experiments using real Internet traces captured by LBL [15] and DATCAT [14]. The SMTP trace captured for 61 minutes, consists of 36,099 total flows with a maximum of 576 concurrent flows. The HTTP trace captured for 61 minutes has 413,371 flows with a maximum of 6,035 active flows. The MIXED trace captured for 10 minutes has a total of 2,491 flows with a maximum of 275 concurrent flows which includes HTTP, SMTP, LDAP and other applications. We used $p_{1}=1, p_{2}=0.1$ for the hybrid approach except for the experiments aimed at confining heavy-hitter flows in which case the probabilities were dynamically adjusted.

\subsection{Experimental Results}

6.3.1. FPR and FNR. To observe the relationship between flow table size and false positives (and false negatives), we conduct the experiments for three traces by varying the flow table as $0.5 X, 2 X$ and $5 X$, where $X$ is the average number of active flows measured using TBP with $T_{\phi}=60$ seconds. Figures 7(a) and 7(b) show the average number of false positives and false negatives for the HTTP trace. From this figure, we can see that false negatives and false positives in CFR and CFR-2 are inversely proportional, while in the case of CFR-LP, they decrease as the size of flow table increases. CFR and CFR-2 does not employ timeout based purging, as a result of which, both the schemes, try to accommodate as many flows as possible unless they are purged by the "clock" activity, which explains an increase (decrease) in false positives (false negatives). Ideally, having low false negatives is desirable, however, lowering false negatives can lead to an increase in false positives. CFR-LP reduces false positive and false negative rates as compared to FCF by $78 \%$ and $73.7 \%$, and it outperforms TBP by $51.1 \%$ and $12.5 \%$ with $2 X$ table size. FCF shows the largest number of false positives due to bucket-level purging and shows only a slight decrease in false negatives under large flow table size e.g., $5 X$. Overall, our CFR approaches are quite accurate irrespective of the dynamics of the traffic mix, outperforming TBP and FCF in terms of FNR.

6.3.2. Estimation of active flows. Average number of active flows $N$ in a trace is given by,

$$
N=\frac{1}{T} \sum_{\theta=1}^{T} N_{\theta},
$$

where $T$ is the number of timeout intervals and $N_{\theta}$ is obtained using equation 4.

Figure 7(c) shows the estimation of average number of active flows $N$ using equation (5), under CFR-LP, TBP and FCF using different flow table sizes. Compared to $N$, TBP overestimates the number of active flows. Among all the purging schemes, CFR-LP is the best estimator of active flows. Also, the experiment showed that FCF is most inaccurate estimator of active flows.

6.3.3. Performance under SYN flooding. To achieve the effect of SYN flood, 3\% spoofed SYN packets which may go undetected were randomly generated and continuously 


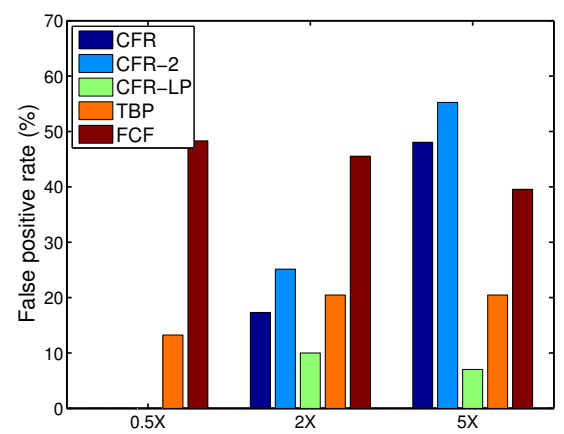

(a) FPR

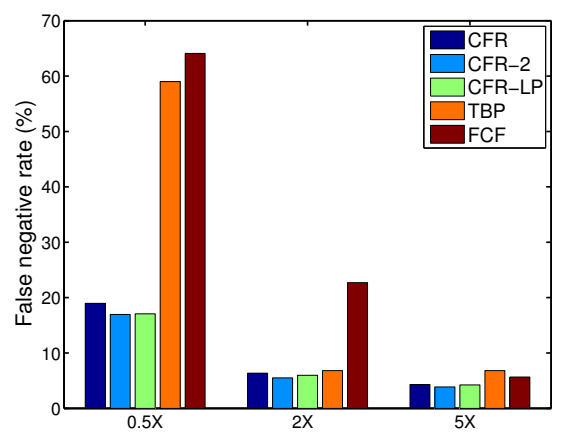

(b) FNR

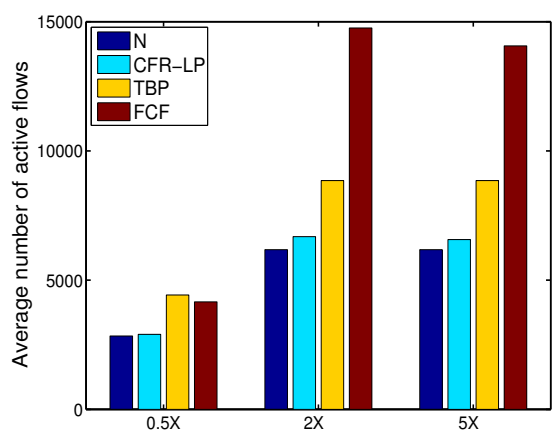

(c) Estimated avg. number of active flows

Figure 7. Comparison of flow management algorithms (HTTP trace, $T_{\theta}=60$ )

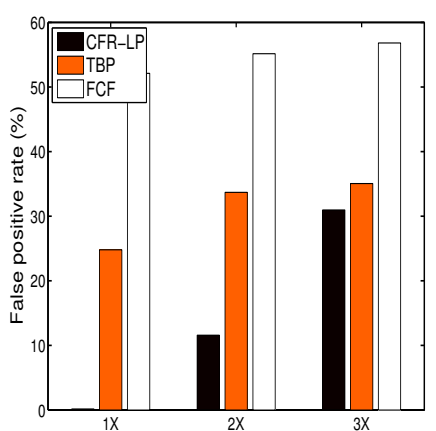

(a) FPR

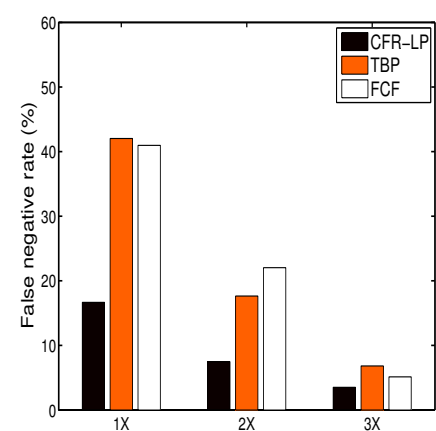

(b) FNR

Figure 8. Comparison of flow management algorithms under 3\% SYN flooding (SMTP trace, $T_{\theta}=30$ )

injected among normal packets of the SMTP trace. FPR and FNR of only normal flows were measured. We observed that 3\% of injected SYN packets increased the number of active flows to more than twice, around 1, 150. This increase may cause an overflow of the flow table when the memory resources are limited in a router. To avoid this, the flow table size should be sufficiently large (at least three times) so as not to miss the active normal flows. However, this enlargement of the flow table comes at a price, especially when using the fast SRAM memory in a router. If flow table size is not sufficiently large, SYN flooding may affect the per-flow monitor. As shown in Figure 8, CFR-LP still performs best even under SYN flooding. We find that our CFR-LP reduces the FPR and FNR of TBP by $65.4 \%$ and $57.6 \%$ and FCF by $79 \%$ and $66 \%$ respectively, when the table size is twice the average number of active flows. This shows that our CFR algorithms are resilient to malicious attacks such as SYN flooding or port scanning.

6.3.4. Confinement of heavy-hitters. To see how our schemes can confine heavy-hitters (i.e., in state $V_{3}$ ), we ran the experiment on the three traces and measured error

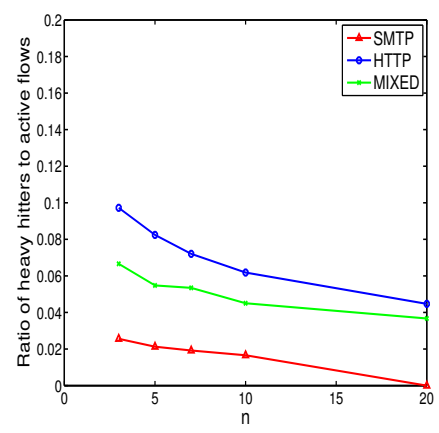

(a) Ratio of heavy-hitters in $V_{3}$ to active flows

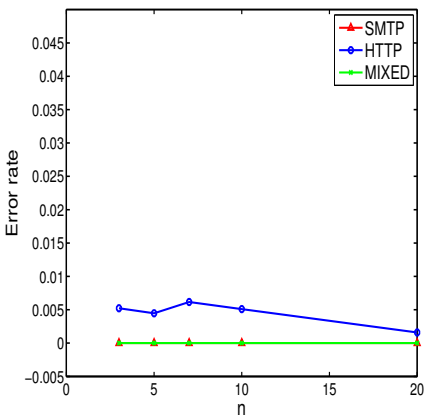

(b) Error rate
Figure 9. Heavy hitter confinement

rate and the ratio of flows in $V_{3}$ to active flows under the CFR-LP scheme. We used parameters of $T_{\theta}=60$ and $p_{1}=0.1 . p_{2}$ is dynamically adjusted as described in equation 2. Figure 9 shows that detected heavy-hitters, i.e., flows in state $V_{3}$ are below $10 \%$ of active flows for all the three traces and the error rate of heavy-hitters is also quite small (less than 0.5\%). This implies that we can monitor only the flows in state $V_{3}$ to detect heavy-hitters without the need to monitor all the flows. Data of these confined flows only can be exchanged for the purpose of coordinated heavy-hitter detection. Therefore, our schemes can help to reduce the overhead of detecting heavy-hitters by reducing the number of flows to be observed.

\section{Conclusions and Future Work}

In this paper, we have proposed a new kind of flow table implementation, based on the simple and efficient replacement policy "clock", and a hybrid approach combining both purging and replacement. Simulation studies have shown that our schemes are robust with respect to maintaining per-flow state irrespective of flow memory size and realistic changes in traffic including malicious attacks. Our approaches can 
accommodate limited hardware and software resources in addition to very high data rates by avoiding flow table overflow and maintaining an ordered list. Thus, the proposed techniques are effectively applicable for high-speed networking devices for enterprise-wide coordination in terms of reduced hardware/software complexity and enhanced realtime processing.

We are currently investigating how to quickly and efficiently migrate and merge flow based data, especially the accuracy of flow discrimination, among multiple flow analyzers in an enterprise, e.g., based on a scheme to reduce the volume of reporting data by clustering the flows according to their frequency. A major concern is that such information sharing could be maliciously induced as part of a defensesoftening pre-attack. Thus, we are carefully considering how to facilitate anomalous high volume of information sharing.

\section{References}

[1] Cisco Systems, Inc., "IOS XR 12000 Router," http://www.cisco.com/en/US/products/ps6342/index.html.

[2] V. Paxson, "Bro: a System for Detecting Network Intruders in Real-time," Computer Netw., vol. 31, no. 23-24, pp. 24352463, 1999.

[3] "Comcast's broadband usage cap won't hurt my mom," http://www.pcmag.com, October 2008.

[4] K. Levchenko, R. Paturi, and G. Varghese, "On the difficulty of scalably detecting network attacks," in Proc. ACM CCS, 2004, pp. 12-20.

[5] F. Bonomi, M. Mitzenmacher, R. Panigrahy, S. Singh, and G. Varghese, "Beyond bloom filters: from approximate membership checks to approximate state machines," in Proc. ACM SIGCOMM, 2006.

[6] S. Yoon, B. koo Kim, J. Oh, and J. Jang, "High Performance Session State Management Scheme for Stateful Packet Inspection," in Proc. APNOMS, 2007, pp. 591-594.

[7] V. Sekar, M. K. Reiter, W. Willinger, H. Zhang, R. R. Kompella, and D. G. Andersen, "cSamp: A system for networkwide flow monitoring," in Proc. 5th USENIX NSDI, 2008.

[8] H. Kim, J.-H. Kim, I. Kang, and S. Bahk, "Preventing Session Table Explosion in Packet Inspection Computers," IEEE Trans. Computers, vol. 54, no. 2, pp. 238-240, 2005.

[9] G. Nam, P. D. Patankar, G. Kesidis, and C. R. Das, "Managing per-flow state of TCP sessions in Internet routers: Session Purging,” Penn State Univ. TR CSE08-120, 2008.

[10] J. Xu and M. Singhal, "Cost-Effective Flow Table Designs for High-Speed Routers: Architecture and Performance Evaluation," IEEE Trans. Comput., vol. 51, no. 9, pp. 1089-1099, 2002.

[11] X. Li, Z. Ji, and M. Hu, "Session Table Architecture for Defending SYN Flood Attack," in Proc. ICICS, 2005.
[12] S. Bansal and D. S. Modha, "CAR: Clock with Adaptive Replacement," in Proc. USENIX FAST, 2004.

[13] A. Kortebi, L. Muscariello, S. Oueslati, and J. Roberts, "Minimizing the overhead in implementing flow-aware networking," in Proc. ANCS, 2005.

[14] "Internet Measurement Data Catalog," http://www.datcat.org/.

[15] "LBNL/ICSI Enterprise Tracing Project," http://www.icir.org/enterprise-tracing/.

[16] Snort, http://www.snort.org/.

[17] "Cisco NetFlow," http://www.cisco.com/web/go/netflow.

[18] C. Estan, K. Keys, D. Moore, and G. Varghese, "Building a better NetFlow," in Proc. ACM SIGCOMM, 2004.

[19] A. Kumar and J. J. Xu, "Sketch Guided Sampling - Using OnLine Estimates of Flow Size for Adaptive Data Collection," in Proc. IEEE INFOCOM, 2006.

[20] A. Ramachandran, S. Seetharaman, N. Feamster, and V. Vazirani, "Fast Monitoring of Traffic Subpopulations," in Proc. ACM IMC, 2008.

[21] G. S. Manku and R. Motwani, "Approximate frequency counts over data streams," in Proc. VLDB, 2002.

[22] C. Estan, G. Varghese, and M. Fisk, "Bitmap algorithms for counting active flows on high-speed links," IEEE/ACM Trans. Netw., vol. 14, no. 5, pp. 925-937, 2006.

[23] H. Song, S. Dharmapurikar, J. Turner, and J. Lockwood, "Fast Hash Table Lookup Using Extended Bloom Filter: An Aid to Network Processing," in Proc. ACM SIGCOMM, 2005.

[24] N. Duffield, C. Lund, and M. Thorup, "Estimating flow distributions from sampled flow statistics," in Proc. ACM SIGCOMM, 2003, pp. 325-336.

[25] Marco, I. Stoica, and H. Zhang, "TCP model for short lived flows," IEEE Communications Letters, vol. 6, no. 2, pp. 8587, 2002.

[26] J. Lemon, "Resisting SYN flood DoS attacks with a SYN cache," in Proc. the BSD Conference, 2002.

[27] T. J. Ott, T. V. Lakshman, and L. H. Wong, "SRED: Stabilized RED,” in Proc. IEEE INFOCOM, 1999, pp. 1346-1355.

[28] S. Yi, X. Deng, G. Kesidis, and C. R. Das, "A Dynamic Quarantine Scheme for Controlling Unresponsive TCP sessions," Telecommunication Systems, vol. 37, no. 4, pp. 169189, 2008.

[29] Y. Lu, B. Prabhakar, and F. Bonomi, "ElephantTrap: A low cost device for identifying large flows," in Proc. HighPerformance Interconnects, 2007, pp. 99-108.

[30] R. Pan, B. Prabhakar, and K. Psounis, "CHOKE, A Stateless Active Queue Management Scheme for Approximating Fair Bandwidth Allocation," in Proc. IEEE INFOCOM, 2000. [Online]. Available: citeseer.ist.psu.edu/article/pan00choke.html

[31] C. Estan and G. Varghese, "New directions in traffic measurement and accounting," in Proc. ACM SIGCOMM, 2002. 